\title{
The cellular localization of autotaxin impacts on its biological functions in human thyroid carcinoma cells
}

\author{
ANJA SEIFERT ${ }^{1}$, THOMAS KLONISCH ${ }^{2}$, JENS WULFAENGER ${ }^{1}$, FRIEDRICH HAAG ${ }^{3}$, \\ HENNING DRALLE ${ }^{4}$, JÜRGEN LANGNER ${ }^{1}$, CUONG HOANG-VU $^{4}$ and ASTRID KEHLEN ${ }^{1,4}$
}

${ }^{1}$ Institute of Medical Immunology, University of Halle-Wittenberg, Halle/Saale, Germany; ${ }^{2}$ Department of Human
Anatomy and Cell Science, University of Manitoba, Winnipeg, Manitoba, Canada; ${ }^{3}$ Institute of Immunology,
University of Hamburg, Hamburg; ${ }^{4}$ Department of Surgery, University of Halle-Wittenberg, Halle/Saale, Germany

Received November 5, 2007; Accepted December 17, 2007

\begin{abstract}
Autotaxin (ATX/NPP2) shows a nucleotide pyrophosphatase/phosphodiesterase and lysophospholipase D (lysoPLD) activity and is a member of a family of structurallyrelated mammalian ecto-nucleotide pyrophosphate/phosphodiesterases (E-NPP1-3). ATX is unique among E-NPP as it is secreted and not membrane-bound as are NPP1 and -3. The ATX gene activity is significantly higher in undifferentiated anaplastic (UTC) as compared to follicular (FTC) and papillary thyroid carcinomas (PTC) or goiter tissues. ATX also enhances the motility of thyroid tumor cells. We bioengineered stable transfectants of the human thyroid carcinoma cell line FTC-238 expressing either bioactively-secreted (sATX) or membrane-anchored ATX (mATX) to identify the biological functions of ATX which critically depend on the E-NPP member being secreted and provide insight into the effects of high local ATX concentrations and cellular responses. An increased cell motility was exclusively observed with FTC-238 sATX transfectants, whereas membraneanchored ATX appeared to impair motility. We identified IL13 as an upstream suppressor of ATX expression in FTC-238, ATX-mediated motility in FTC-238 and stable transfectants, with IL-1ß having the strongest motility-suppressive effect on FTC-238 sATX clones. sATX and mATX strongly increased the anchorage-independent colony formation of
\end{abstract}

Correspondence to: Dr Astrid Kehlen, Martin Luther University Halle-Wittenberg, Universitätsklinik und Poliklinik für Allgemein-, Viszeral- und Gefässchirurgie, ECHO Labor, Magdeburger Str. 18, D-06097 Halle, Germany

E-mail: astrid.kehlen@medizin.uni-halle.de

Abbreviations: ATX, autotaxin; lysoPLD, lysophospholipase D; LPC, lysophosphatidylcholine; LPA, lysophosphatidic acid; PTC, papillary thyroid carcinoma; FTC, follicular thyroid carcinoma; UTC, undifferentiated thyroid carcinoma

Key words: autotaxin, lysophospholipase D, thyroid carcinoma, cancer-testis antigen, BAGE
FTC-238 but the size and number of colonies formed in the soft agar were significantly smaller in FTC-238 mATX versus the FTC-238 sATX clones. The cancer-testis antigen BAGE was identified as a novel target gene of ATX in FTC238. Transcript levels for BAGE were 6-fold higher in FTC238 mATX versus sATX clones. Increased BAGE transcript levels were also detected in tissues of patients with UTC versus FTC, PTC or goiter tissues. In summary, enhanced tumor cell motility and tumorigenic capacity critically depended on sATX in thyroid carcinoma cells. Irrespective of its compartmentalization, the cancer-testis antigen BAGE was identified as a novel target gene of ATX in FTC-238 and a potential new tissue marker in UTC tissues, which we had previously shown to express high levels of ATX.

\section{Introduction}

Autotaxin (ATX/NPP2) is a $125 \mathrm{kDa}$ glycoprotein and was originally isolated from the conditioned medium of human melanoma A2058 cells (1). The sequence analysis of ATX showed significant homology with a family of cell surface type I phosphodiesterases. ATX has been classified as a member of the ecto-nucleotide pyrophosphate/phosphodiesterase family (E-NPP) $(2,3)$. ATX was found to possess a lysophospholipase D (lyso-PLD) activity which results in the hydrolysis of lysophosphatidyl choline (LPC) to the lysophosphatidic acid (LPA) $(4,5)$. ATX has the capacity to hydrolyse several other glycerophospholipids to produce LPA, including lysophosphatidylserine, lysophosphatidylethanolamine and lysophosphatidylinositol (6). Three structurally-related mammalian E-NPPs have been studied in more detail: NPP1 (PC-1), NPP2 (ATX and PD-I $\alpha$ ) and NPP3 (gp130 RB 13-6, PD-Iß and B10). The E-NPP members show a 40-50\% identity in their 800 residue extracellular domains. While ATX/NPP2 is a secreted protein, NPP1 and -3 are anchored in the cell membrane by a transmembrane domain followed by a short N-terminal intracellular domain of $\sim 20$ residues $(7,8)$. The secretion of ATX involves the cleavage of a 27 amino acid signal sequence by signal peptidase and further proteolytic maturation by pro-protein convertases.

ATX stimulates random and directed motility in a variety of tumor cell lines, including those from breast, renal and 
Table I. Sequence of primers used for cloning, standard primer construction and PCR amplification.

\begin{tabular}{lll}
\hline Gene & No. & \multicolumn{1}{c}{ Sequence 5' $^{\prime} 3^{\prime}$} \\
\hline ATX & 1 & GATTTAGGTGACACTATAGAATACGTTGCAAGGAAACCTTTGGA \\
& 2 & AACTTCCTCTGGCATGGTTG \\
& 3 & GTTGCAAGGAAACCTTTGGA \\
& 4 & ATATTTCTCGAGATGGCAAGGAGGAGCTCGTT \\
BAGE & 5 & ATATTTGGATCCAATCTCGCTCTCATATGTATGCAGGT \\
& 1 & GATTTAGGTGACACTATAGAATAGAGGCTGGAGCCTGTAACAC \\
& 2 & GAGGCTGGAGCCTGTAACAC \\
\hline
\end{tabular}

thyroid carcinoma, melanoma and neuroblastoma $(1,9-12)$. ATX augments the tumorigenic capacity and metastatic potential of ras-transformed cells and appears to act as an angiogenic factor $(13,14)$. We recently quantified the ATX mRNA expression in thyroid carcinoma cell lines and in tissues of patients with thyroid carcinomas. The ATX gene activity was significantly higher in UTC cell lines and tumor tissues as compared to FTC cell lines, FTC tissues or goiter tissues used as a control. FTC-133 stable transfectants overexpressing a soluble ATX showed a higher lysoPLD activity, enhanced proliferation and an increased migratory activity. ATX also showed a paracrine stimulatory effect on the motility of other thyroid carcinoma cell lines (9). When expressed in FTC-133 or COS-1 cells, ATX was mainly found in the supernatant, whereas NPP1 was abundant in cell lysates $(9,15)$.

In the present study, we identified functions of ATX which critically depend on the E-NPP member being secreted. To address this question, we generated stable transfectants of the human thyroid carcinoma cell line FTC-238 expressing either bioactively-secreted (sATX) or membrane-anchored ATX (mATX). The cancer-testis antigen BAGE was identified as a novel target gene of ATX in FTC-238 and, as with ATX, BAGE was found to be up-regulated in human UTC tissues.

\section{Materials and methods}

Thyroid tissues and the FTC-238 cell line. A total of 40 thyroid tissues, including 10 follicular (FTC), 10 papillary (PTC) and 10 undifferentiated thyroid carcinomas (UTC) and 10 goiter tissues were obtained from patients undergoing clinicallyindicated surgery and stored at $-80^{\circ} \mathrm{C}$ until use. This study was approved by the local university ethics committee and all patients gave their written consent prior to surgery. Each tumor was scored according to the pTNM classification (16). The human thyroid cancer cell line FTC-238 (17) was cultured in RPMI-1640 medium and $10 \%$ fetal calf serum (FCS) was added at $37^{\circ} \mathrm{C}$ in a $\mathrm{CO}_{2}$ incubator. To analyze the effect of the localization of ATX within human thyroid carcinoma cells on cellular functions, we transfected FTC-238 cells with the pEGFP-C3/ATX construct or the pD38tm/ATX vector which contains the intracellular and transmembrane domains (aa 1-62) of mouse CD38. Stable transfectants with the pEGFP-C3/ATX construct (FTC-238 sATX) secreted
ATX as a soluble form in the cell culture supernatant. FTC238 transfected with the pD38tm/ATX vector (FTC-238 mATX), produced membrane-anchored ATX. FTC-238 transfected with the pEGFP construct (FTC-238 GFP) or pD38tm vector (FTC-238 pD38tm) which served as mock controls.

RNA isolation and cDNA synthesis. RNA isolation and reverse transcription have recently been described (9). Briefly, a first strand cDNA synthesis was performed according to the manufacturer's instructions using $500 \mathrm{ng}$ total RNA, $50 \mathrm{pmol}$ of random primer (Roche, Mannheim, Germany), and $0.5 \mu 1$ (200 U/ $\mu 1)$ Superscript ${ }^{\text {TM }}$ II-RT (Invitrogen, Karlsruhe, Germany). For quantitative RT-PCR (Q-RT-PCR), six dilutions of standard and total RNA, respectively, were converted into cDNA in separate tubes.

The construction of RNA standards. The RNA standards were constructed as previously described (18). Briefly, for the construction of standard RNA, composite primers were synthesized for ATX and BAGE (Table I). The forward primer 1 contained a 5'-SP6 RNA polymerase binding site (Table I, underlined) followed by the specific sequence of the appropriate gene to be amplified. The PCR product obtained with primers 1 and 2 was gel-purified (QIA quick gel extraction kit, Qiagen, Hilden, Germany) followed by in vitro transcription from the SP6 promoter (Roche). The RNA copy was quantified at $260 \mathrm{~nm}$ and used as the standard in the quantitative RT-PCR reaction.

Quantitative PCR (Q-RT-PCR) analysis. For quantitation, $1 \mu 1$ of the reverse transcriptase reaction mixture was added to a $20 \mu 1$ reaction mixture of the QuantiTect SYBR-Green PCR kit (Qiagen) and $0.5 \mu \mathrm{M}$ from primers 2 and 3 (Table I). A negative control containing the complete MasterMix without a DNA template was included. Samples of the standard and sample cDNA, respectively, were run in triplicate on the Rotor-Gene 2000 (LTF, Wasserburg, Germany). Initial denaturation at $95^{\circ} \mathrm{C}$ for $900 \mathrm{sec}$ was followed by 40 cycles with denaturation at $95^{\circ} \mathrm{C}$ for $15 \mathrm{sec}$, annealing at $60^{\circ} \mathrm{C}$ for $30 \mathrm{sec}$ and extension at $72^{\circ} \mathrm{C}$ for $20 \mathrm{sec}$. The fluorescence intensity of SYBR-Green, specifically incorporated in the double-stranded DNA amplicon reflecting the amount of formed PCR product, was read after each extension step at 
A

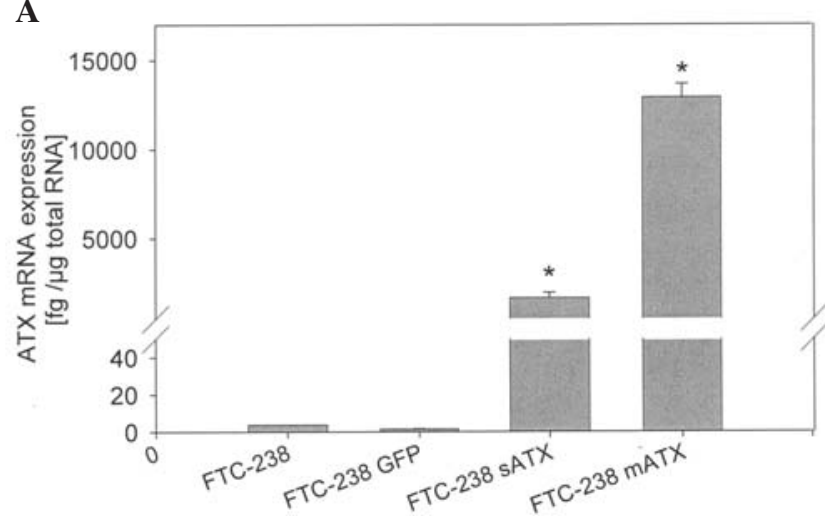

B

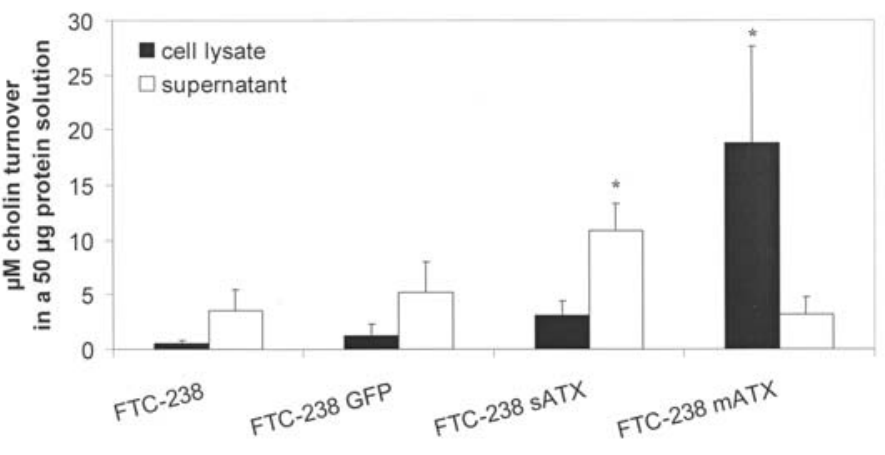

Figure 1. (A) Q-RT-PCR analysis of the absolute amounts of the ATX mRNA levels in FTC-238 and FTC-238 GFP, sATX and mATX transfectants (n=4, $\left.{ }^{*} \mathrm{P}<0.001\right)$. (B) The lysoPLD activity of non-transfected and FTC-238 GFP, sATX and mATX clones $\left(\mathrm{n}=4{ }^{*} \mathrm{P}<0.05\right)$. The highest ATX gene and lysoPLD activity were observed in FTC-238 mATX.

$72^{\circ} \mathrm{C}$. For verification of the PCR, product melting curves were generated and single amplicons were confirmed by agarose gel electrophoresis. Absolute RNA amounts were determined with the Rotor-Gene software version 4.6 in quantitation mode.

The generation of stable transfectants overexpressing human ATX. The full-length cDNA of the human ATX was cloned in the pEGFP-C3 vector (BD Clontech, Heidelberg, Germany) using the primers ATX4 and -5 (Table I). To express ATX as a membrane-anchored protein, the extracellular domain (aa 57-863) of the human ATX, carrying a C-terminal V5 epitope tag, was cloned into the pD38tm vector. FTC-238 $\left(5 \times 10^{5}\right.$ cells) was transfected with $1.5 \mu \mathrm{g}$ of either the pEGFP-C3 empty vector, the pEGFP-C3/ATX construct, the pD38tm empty vector, or the pD38tm/ATX construct using $3 \mu 1$ Lipofectamine 2000 (Invitrogen) according to the manufacturer's instructions. Selection was initiated $48 \mathrm{~h}$ after transfection by adding $75 \mu \mathrm{g} / \mathrm{ml} \mathrm{G} 418$ (Invitrogen). The selection medium was changed every 4 days and G418resistant cell clones were isolated and expanded for further characterization.

The soft agar assay. Cell transfectants $\left(5 \times 10^{4}\right.$ cells $)$ were seeded in RPMI containing $0.6 \%$ agar and $20 \%$ FCS in a 6-well plate, overlaid and cultured with RPMI and $10 \%$ $\mathrm{FCS}$ at $37^{\circ} \mathrm{C}$ in a $\mathrm{CO}_{2}$ incubator for 1 month. The medium was changed every 2-3 days (19). Colonies were stained with iodonitrotetrazolium chloride (Sigma) and colony numbers and sizes per filter were determined by counting four powered (x20) magnification fields under bright field microscopy (Axioplan microscope with Axiovision 2.0.1 software).

The lysoPLD enzyme assay. Quantitation of the lysoPLD activity was previously described (9). Briefly, the concentrated cell culture supernatant or the cell pellet was incubated for $12 \mathrm{~h}$ with $2 \mathrm{mM} \mathrm{LPC} \mathrm{(Sigma)} \mathrm{in} 100 \mathrm{mM}$ Tris- $\mathrm{HCl}$ at $\mathrm{pH} 9.0$, $5 \mathrm{mM} \mathrm{MgCl}{ }_{2}$ and $500 \mathrm{mM} \mathrm{NaCl}$. Released choline was detected by an enzymatic photometric method using $0.5 \mathrm{U}$ choline oxidase, $0.02 \mathrm{U}$ peroxidase, $0.05 \mathrm{M}$ aminoantipyrene and 0.21 M phenol (all from Sigma) in a reaction volume of $100 \mu 1$. Absorbance was read at $500 \mathrm{~nm}$ after $30 \mathrm{~min}$ at $37^{\circ} \mathrm{C}$.

The cell motility assay. The cell motility assay was previously described (9). Briefly, the cellular motility of ATXtransfected FTC-238 was evaluated in 24-well transwell chambers (Costar, Bodenheim, Germany), with upper and lower culture compartments being separated by polycarbonate filters with a pore size of $8 \mu \mathrm{m}$. In autocrine motility assays, $1 \times 10^{5}$ cells/well were seeded in RPMI containing $0.1 \%$ BSA onto the filters and incubated for $24 \mathrm{~h}$. For stimulation experiments, cells were serum-starved for 1 day and treated with recombinant IL-1ß (all $10 \mathrm{ng} / \mathrm{ml}$, Strathmann Biotech, Hamburg, Germany) prior to seeding cells on the filters. After migration for $24 \mathrm{~h}$, the cells on top of the membrane were removed, and transfectants that had traversed the membrane pores and were located at the lower surface were stained with toluidine-blue. The migrated cells were counted in triplicate under light microscopy (four power fields/filter).

Statistical analysis. Data are expressed as mean \pm SD. All statistical analyses were performed using a Student's t-test.

\section{Results}

Increased cell motility of the human thyroid carcinoma cells requires the secretion of ATX. By employing the follicular thyroid carcinoma cell line FTC-238, stable transfectants generated overexpressing - secreted (FTC-238 sATX) and membrane-anchored ATX (FTC-238 sATX). Real-time RTPCR showed a significantly enhanced expression of sATX and mATX mRNA in the clones, whereas the mock control cells and untransfected FTC-238 showed a very low endogenous ATX expression (Fig. 1A). FTC-238 sATX and mATX transfectants produced a bioactive ATX. The supernatant of FTC-238 sATX clones contained a high lysoPLD activity indicating the secretion of a functional ATX by these clones (Fig. 1B). As with FTC-238 and the mock cells, FTC-238 sATX displayed low levels of lysoPLD activity in the cell lysates suggesting that most of the ATX produced was 


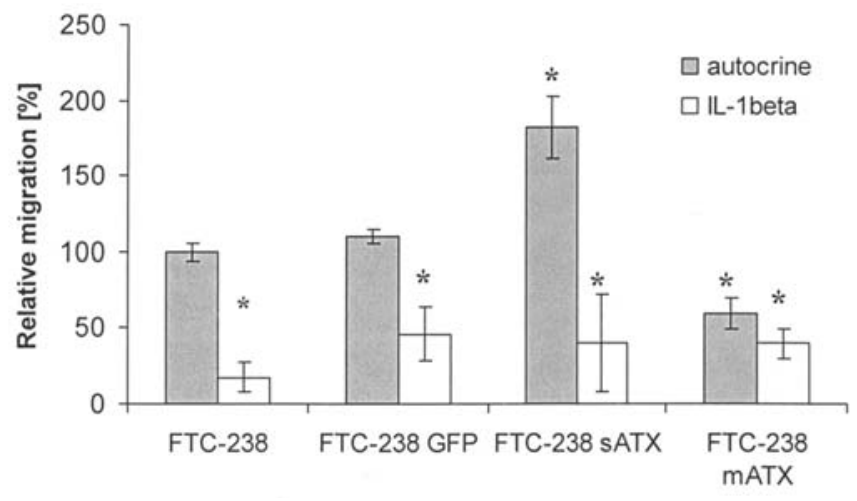

Figure 2. The autocrine effect of ATX on the migration of FTC-238. FTC-238 GFP, FTC-238 sATX and mATX transfectants and untransfected FTC-238 were seeded in a cell density of $1 \times 10^{5}$ cells/well and were incubated with or without IL-1ß added to the upper compartment of a transwell chamber. Thyroid carcinoma cells that had traversed the membrane after $24 \mathrm{~h}$ were stained with toluidine blue and counted. The mean \pm SD of four separate experiments is shown, ${ }^{*} \mathrm{P}<0.05$.

released into the supernatant by these transfectants. In contrast, the FTC-238 mATX transfectants showed a high lysoPLD enzymatic activity in cell lysates but negligible levels of ATX in the supernatant (Fig. 1B). We employed a transwell filter assay to determine the motility of FTC-238 transfectants. The motility was significantly enhanced for the FTC-238 sATX clones as compared to the FTC-238 mATX, mock and parental FTC-238 (Fig. 2). These data extend our previous findings in FTC-133 transfectants which overexpress and secrete the human ATX (9). FTC-238 mATX transfectants showed the lowest motility of all cells (Fig. 2), despite a high ATX mRNA expression and lysoPLD activity (Fig. 1). Thus, the motility-enhancing quality of ATX was a paracrine effect depending on the nucleotide pyrophosphatase/phosphodiesterase to be secreted. The addition of IL-1ß to the culture medium caused a significant decrease in tumor cell motility in all FTC-238 transfectants and parental cells and this motilitysuppressive effect was most pronounced in FTC-238 sATX transfectants (Fig. 2). This reduction in FTC-238 motility in the presence of IL-1B coincided with a $35 \%$ reduction in the ATX mRNA expression in FTC-238 (data not shown) and confirmed our previous result of an IL-1ß-induced downregulation of the ATX gene activity in FTC-133 (9).

ATX increases the tumorigenic capacity of human thyroid carcinoma cells. We investigated the ability of sATX and mATX to enhance the oncogenic potential of FTC-238 by determining the anchorage-independent growth of the FTC238 sATX and mATX clones in colony soft agar assays. Parental and mock control cells generated small-sized colonies of $\sim 20 \mu \mathrm{m}$ in diameter (Fig. 3A). FTC-238 mATX formed colonies of 100-150 $\mu \mathrm{m}$ in diameter (Fig. 3A) and the number of colonies were $\sim 12$-fold more than in the mock controls (Fig. 3B). FTC-238 sATX transfectants generated the largest colonies with a diameter of 300-400 $\mu \mathrm{m}$ (Fig. 3A) and exceeded (3-fold) the number of colonies counted with FTC-238 mATX (Fig. 3B). Thus, although sATX and mATX increased the anchorage-independent colony growth of FTC-238, naturally-occurring secreted ATX, rather than its engineered membrane-bound form, appeared to be significantly more efficient in enhancing the tumorigenic capacity of FTC-238 (Fig. 3B).

BAGE is a novel target gene of ATX. Real-time RT-PCR showed BAGE to be a novel ATX target gene in the stable FTC-238 transfectants. BAGE is a cancer-testis antigen and a member of the tumor-associated antigens. Although the FTC-238 mATX and sATX clones showed an up-regulation of BAGE, FTC-238 mATX contained markedly higher levels of BAGE expression (58-fold) as compared to FTC-238 sATX (9-fold) or the mock cells and untransfected FTC-238 which showed a negligible expression of BAGE (Fig. 4). We had previously demonstrated an enhanced ATX expression in human UTC tissues (9). To determine whether human thyroid tissues also displayed an up-regulation in BAGE, the same tissues of patients with thyroid carcinomas used in our previous study were quantified for mRNA expression of BAGE. Median BAGE mRNA levels were highest in the UTC tissues at $7.1 \mathrm{fg} / \mu \mathrm{g}$ total RNA, but significantly lower in PTC at $2.1 \mathrm{fg} / \mu \mathrm{g}$ total RNA, FTC at $1.6 \mathrm{fg} / \mu \mathrm{g}$ total RNA and goiter at $1.5 \mathrm{fg} / \mu \mathrm{g}$ total RNA (Fig. 5). Therefore, we found that an enhanced expression of sATX and BAGE coincides in human UTC.

\section{Discussion}

In the present study, we generated FTC-238 stable transfectantexpressing secreted (FTC-238 sATX) human ATX and a bioengineered membrane-anchored ATX (FTC-238 mATX) to address i) whether the secretion of ATX is a prerequisite for its growth- and motility-promoting actions, and, ii) to determine the tumor cell responses to high local ATX concentrations such as those in mATX. ATX is a unique family member of ecto-nucleotide pyrophosphate/phosphodiesterase (E-NPP1-3). Contrary to the type II transmembrane ectoenzymes NPP1 and -3 , with a short cytoplasmatic tail and a large catalytic ectodomain membrane-bound, ATX (NPP2) is not associated with the plasma membrane but appears to be localized in cytosolic vesicles (20). ATX is synthesized as a preproenzyme and, upon removal of a 27-residue signal peptide, is subsequently cleaved by pro-protein convertases prior to secretion $(7,8)$. The aim of the study was to specify the biological functions that are specific to the secreted ATX, whereas other functions may be independent of the cellular localization of ATX. Notably, we found that the cellular localization of ATX had a marked impact on the ability of the E-NPP member to affect the motility of human thyroid carcinoma cells. As with the FTC-133 sATX clones (9), the secreted ATX caused significant enhancement in the motility of FTC-238 sATX clones. In contrast, despite a higher ATX mRNA expression and an increased lysoPLD activity by the FTC-238 mATX, these transfectants failed to display increased motility. The inability of FTC-238 mATX to increase tumor cell motility was not the result of biologicallyinactive membrane-bound ATX. These clones displayed high levels of lysoPLD enzymatic activity in the cell lysates but not in the supernatant indicating a cell-bound mATX and negligible shedding of membrane-anchored ATX by the FTC-238 mATX transfectants. Although varied glycosylation 
$\mathbf{A}$

FTC-238
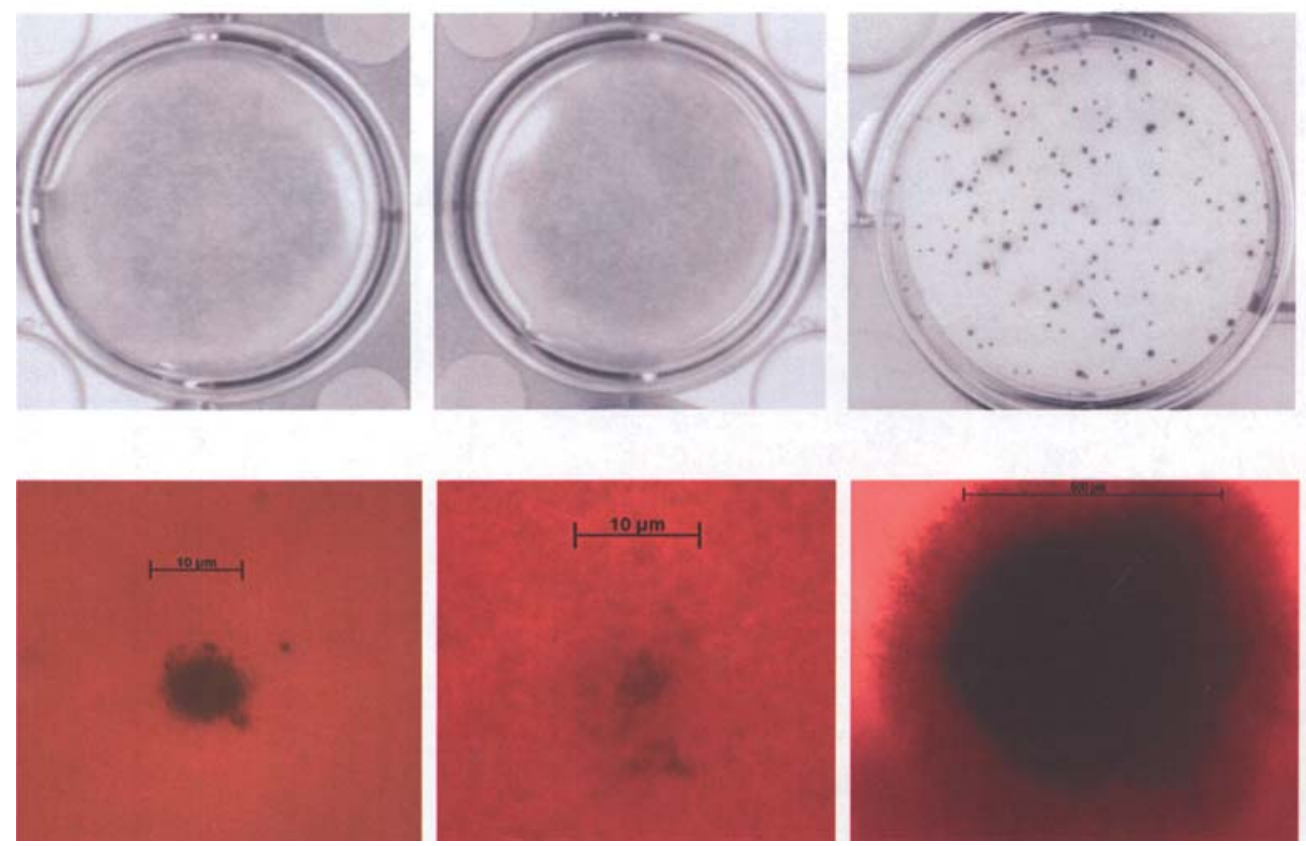

FTC-238 sATX

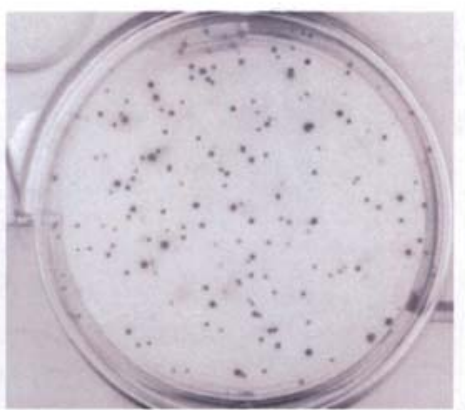

B
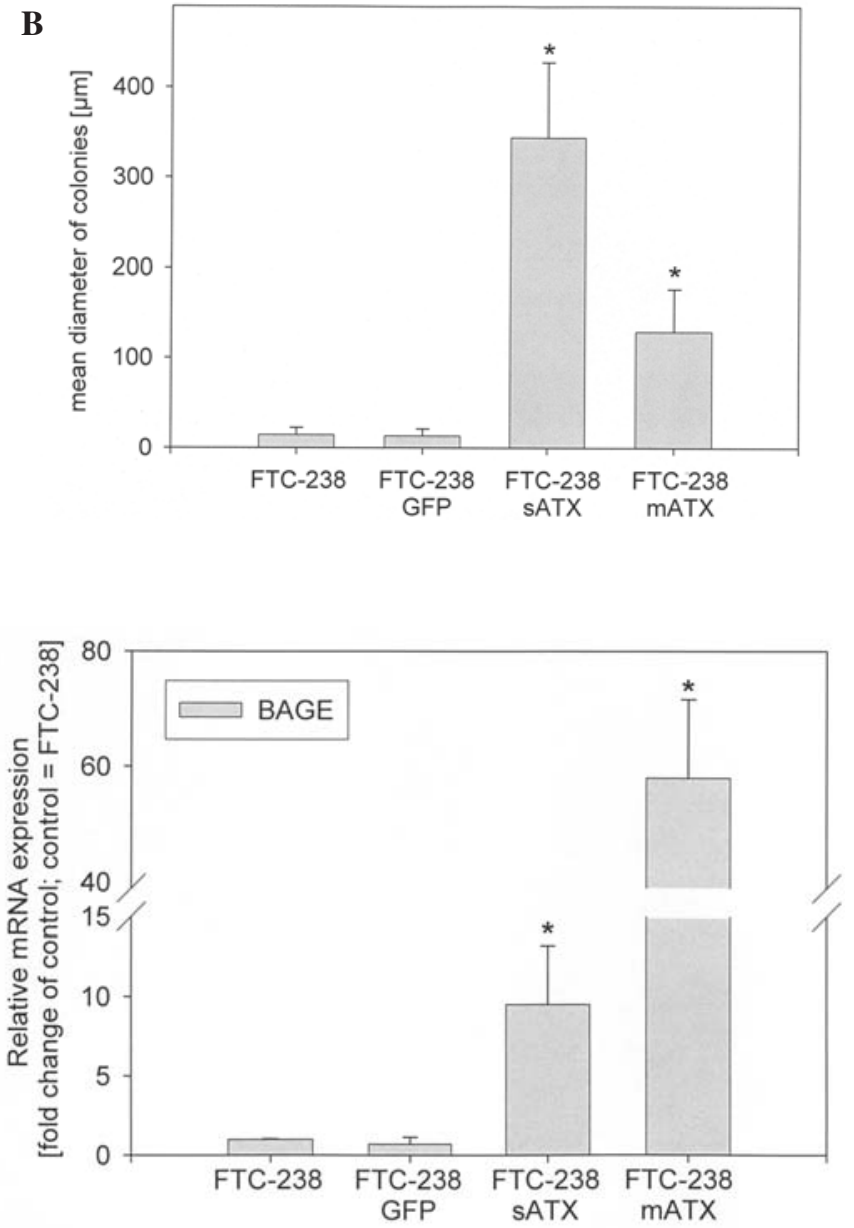

Figure 4. Results of Q-RT-PCR analysis on the effect of ATX on the BAGE gene expression in FTC-238, FTC-238 GFP, FTC-238 sATX and mATX transfectants. Data are given as a fold change of the control (untransfected FTC-238 $=1.0$ ) from four independent experiments. "Statistically significant differences from the control, ${ }^{*} \mathrm{P}<0.01$.

\section{FTC-238 mATX}
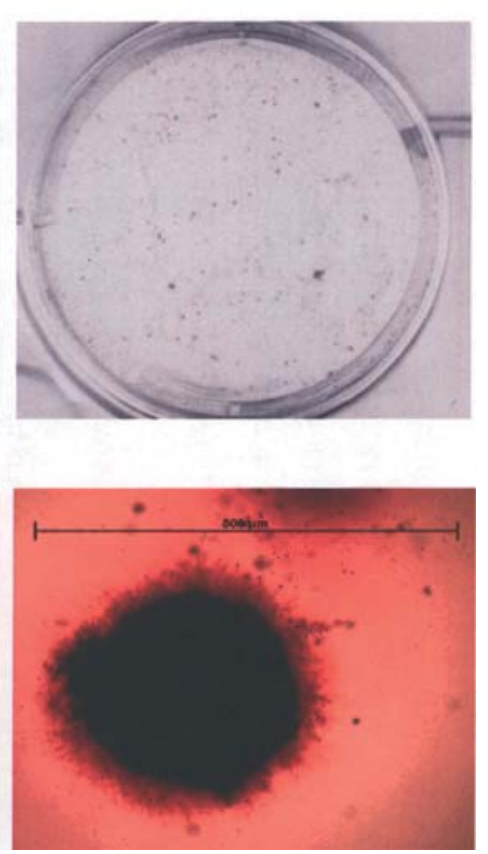

Figure 3. (A) ATX promotes colony formation in soft agar assays. FTC-238 GFP, FTC-238 sATX and mATX transfectants were plated in semi-solid agar medium and the sizes of formed colonies were determined by phasecontrast microscopy (upper panel). Images represent the stained colonies in soft agar plates (lower panel). Images represent the colony growth in soft agar, shown for the untransfected FTC-238 and and FTC-238 GFP mock controls with a magnification bar at $10 \mu \mathrm{m}$. Note the magnification scale at $500 \mu \mathrm{m}$ in the pictures with FTC-238 sATX and mATX transfectants indicating much larger colonies with FTC-238 when exposed to ATX. (B) Number of colonies formed. Data are calculated as mean \pm SD of at least two independent experiments, ${ }^{*} \mathrm{P}<0.001$.

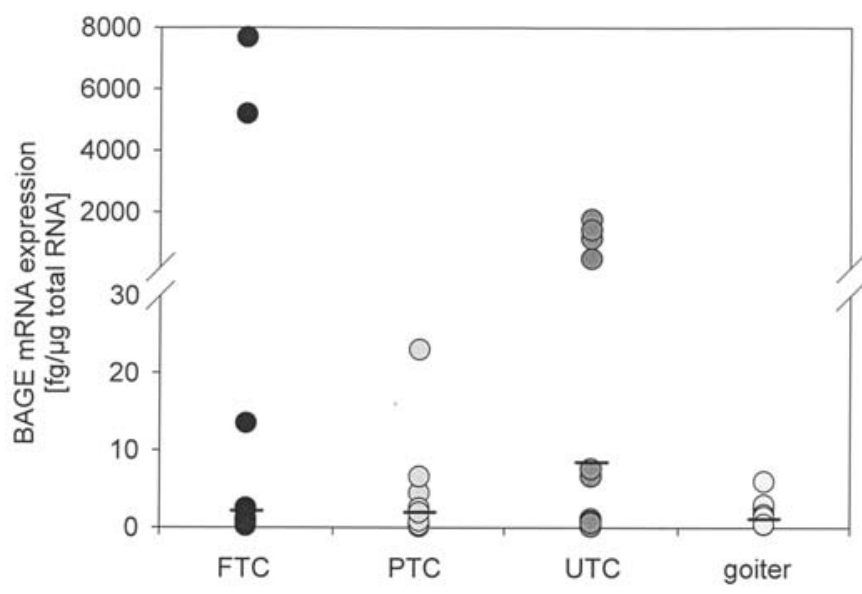

Figure 5. The Q-RT-PCR analysis of the absolute BAGE mRNA amounts in tissues of patients with follicular (FTC), papillary (PTC), undifferentiated (UTC) thyroid carcinoma and goiter tissue, respectively (each patient group: $\mathrm{n}=10$, measured in triplicates; bars represent the median). 
patterns of ATX have been shown to affect the ATX function (7), this possibility is unlikely to explain the differences in motility elicited by sATX and mATX since the two ATX constructs contain the same potential glycosylation sites and were expressed in the same thyroid carcinoma cells.

Secreted ATX exerts its ecto-nucleotide pyrophosphate/ phosphodiesterase and lysophospholipase D activity on the surrounding cells and tissues. We recently demonstrated the oncogenic potential of ATX as an enhancer of proliferation and migration of human thyroid carcinoma cells (9). Studies in athymic nude mice demonstrated that ras-transformed NIH3T3 cells with ATX expression displayed amplified tumorigenic capacity (13). In the pathogenesis of thyroid carcinomas, somatic Ras mutations appear to be an early event and are frequently observed in follicular thyroid carcinomas and cell lines (21). Thus, the combined effect of the Ras mutation and ATX overexpression may contribute to an amplified metastatic potential of thyroid carcinoma cells and suggests an important role for ATX as a promoter of thyroid carcinogenesis and metastasis. In line with these findings and irrespective of its cellular localization, ATX significantly enhanced the oncogenic transforming potential of FTC-238 as shown by the increased anchorage-independent growth in soft agar assays of the two FTC-238 sATX and mATX transfectants. However, consistent and distinct differences in the behavior of FTC-238 sATX and mATX clones were observed. Secreted ATX appeared to promote increased numbers of very large colonies displayed with a dense cellular core surrounded by more loosely arranged cells in the periphery consistent with migratory cells. In contrast, FTC-238 mATX created significantly less and very dense colonies with few peripheral cells, which was in line with the reduced motility observed with FTC-238 mATX. Thus, sATX was more efficient than mATX in conferring a higher oncogenictransforming potential onto FTC-238.

The pro-inflammatory cytokine IL-1B is an important regulator of the ATX expression in thyroid cells and we observed an IL-1ß down-regulated endogenous ATX gene activity in FTC-238 and FTC-133 by 35 and 50\%, respectively (9). In primary thyroid cell cultures, IL-1ß was reported to induce apoptosis by inhibiting adenylate cyclase and ERK1/2 activity (22). Herein we showed that IL-1ß also acts as a powerful inhibitor of cell motility in FTC-238 and this IL-1ßmediated motility-suppressive action was most pronounced in FTC-238 sATX. The present finding of ATX being a novel IL-1ß target gene may provide the basis for novel therapeutic strategies involving the cytokine-mediated suppression of thyroid carcinoma invasiveness and metastasis, particularly in UTC.

We identified the cancer-testis antigen BAGE as a novel ATX target gene in the transformed FTC-238 ATX transfectants. Cancer-testis antigens of the MAGE, BAGE and GAGE family represent a unique class of tumor antigens which are expressed in a variety of carcinomas and are silent in normal tissues, except in testis. The BAGE family contains 15 nearly identical sequences located in the juxta-centromeric regions of chromosomes $9,13,18$ and 21. BAGE encodes for a tumor-associated antigen of as yet unknown function which is recognized by cytotoxic $\mathrm{T}$ lymphocytes in conjunction with MHC class I molecules of various haplotypes on the tumor cell surface. BAGE was shown to be expressed in thyroid medullary and papillary carcinoma, and papillary hyperplasia of the thyroid $(23,24)$. The highest BAGE transcript levels were detected in the FTC-238 mATX transfectants suggesting that higher local mATX concentrations and cellular lysoPLD activity can trigger a differential gene expression in tumor cells. Despite the fact that our cell model of membrane-anchored ATX is a synthetic product shown not to exist naturally, this model may provide a unique insight into the differential cellular responses to high local ATX concentrations within solid thyroid tumor tissues where varying local ATX tissue concentrations are expected to occur. The up-regulation of the BAGE gene expression in FTC-238 cells as a result of ATX overexpression was also observed in tissues of patients with UTC. We had previously detected the highest levels of ATX in UTC versus FTC, PTC and goiter tissues and identified the ecto-nucleotide pyrophosphate/phosphodiesterase as a novel tissue marker for UTC (9).

In conclusion, our thyroid tumor cell models showed the common and distinct cellular responses elicited by secretedand membrane-anchored ATX. The proper processing of ATX from its membrane-bound proform to the secreted product was an essential prerequisite for the motility-enhancing property of ATX. Thus, a distinct and important purpose of the soluble ATX/NPP2 ecto-nucleotide pyrophosphate/ phosphodiesterase activity appears to be the propagation of cellular motility/migration, a functional quality lacking in mATX.

\section{Acknowledgements}

We are grateful to K. Hammje, I. Peters and C. Hamann for excellent technical support. This study was supported by the 'Wilhelm-Roux-Programm', Medical Faculty, Martin Luther University Halle-Wittenberg.

\section{References}

1. Stracke ML, Krutzsch HC, Unsworth EJ, et al: Identification, purification, and partial sequence analysis of autotaxin, a novel motility-stimulating protein. J Biol Chem 267: 2524-2529, 1992.

2. Clair T, Lee HY, Liotta LA and Stracke ML: Autotaxin is an exoenzyme possessing 5'-nucleotide phosphodiesterase/ATP pyrophosphatase and ATPase activities. J Biol Chem 272: 996-1001, 1997

3. Murata J, Lee HY, Clair T, et al: cDNA cloning of the human tumor motility-stimulating protein, autotaxin, reveals a homology with phosphodiesterases. J Biol Chem 269: 30479-30484, 1994.

4. Umezu-Goto M, Kishi Y, Taira A, et al: Autotaxin has lysophospholipase D activity leading to tumor cell growth and motility by lysophosphatidic acid production. J Cell Biol 158: 227-233, 2002.

5. Tokumura A, Majima E, Kariya Y, et al: Identification of human plasma lysophospholipase D, a lysophosphatidic acidproducing enzyme, as autotaxin, a multifunctional phosphodiesterase. J Biol Chem 277: 39436-39442, 2002.

6. Aoki J, Taira A, Takanezawa Y, et al: Serum lysophosphatidic acid is produced through diverse phospholipase pathways. J Biol Chem 277: 48737-48744, 2002.

7. Jansen S, Stefan C, Creemers JW, et al: Proteolytic maturation and activation of autotaxin (NPP2), a secreted metastasisenhancing lysophospholipase D. J Cell Sci 118: 3081-3089, 2005.

8. Koike S, Keino-Masu K, Ohto T and Masu M: The N-terminal hydrophobic sequence of autotaxin (ENPP2) functions as a signal peptide. Genes Cells 11: 133-142, 2006. 
9. Kehlen A, Englert N, Seifert A, et al: Expression, regulation and function of autotaxin in thyroid carcinomas. Int $\mathrm{J}$ Cancer 109: 833-838, 2004.

10. Yang SY, Lee J, Park CG, et al: Expression of autotaxin (NPP-2) is closely linked to invasiveness of breast cancer cells Clin Exp Metastasis 19: 603-608, 2002.

11. Kawagoe H, Stracke ML, Nakamura H and Sano K: Expression and transcriptional regulation of the PD-Ialpha/autotaxin gene in neuroblastoma. Cancer Res 57: 2516-2521, 1997.

12. Yang Y, Mou L, Liu N and Tsao MS: Autotaxin expression in non-small-cell lung cancer. Am J Respir Cell Mol Biol 21: 216-222, 1999.

13. Nam SW, Clair T, Campo CK, et al: Autotaxin (ATX), a potent tumor motogen, augments invasive and metastatic potential of ras-transformed cells. Oncogene 19: 241-247, 2000.

14. Nam SW, Clair T, Kim YS, et al: Autotaxin (NPP-2), a metastasis-enhancing motogen, is an angiogenic factor. Cancer Res 61: 6938-6944, 2001.

15. Gijsbers R, Aoki J, Arai H and Bollen M: The hydrolysis of lysophospholipids and nucleotides by autotaxin (NPP2) involves a single catalytic site. FEBS Lett 538: 60-64, 2003.

16. Kehlen A, Lendeckel U, Dralle H, et al: Biological significance of aminopeptidase N/CD13 in thyroid carcinomas. Cancer Res 63: 8500-8506, 2003.

17. Demeure MJ, Damsky CH, Elfman F, et al: Invasion by cultured human follicular thyroid cancer correlates with increased beta 1 integrins and production of proteases. World J Surg 16: 770-776, 1992.
18. Kehlen A, Thiele K, Riemann D and Langner J: Expression, modulation and signalling of IL-17 receptor in fibroblast-like synoviocytes of patients with rheumatoid arthritis. Clin Exp Immunol 127: 539-546, 2002.

19. Peri A, Bonaccorsi L, Muratori M, et al: Uteroglobin reverts the transformed phenotype in the endometrial adenocarcinoma cell line HEC-1A by disrupting the metabolic pathways generating platelet-activating factor. Int J Cancer 88: 525-534, 2000.

20. Moolenaar WH, van Meeteren LA and Giepmans BN: The ins and outs of lysophosphatidic acid signaling. Bioessays 26 : 870-881, 2004.

21. Gimm O: Thyroid cancer. Cancer Lett 163: 143-156, 2001

22. El Btaouri H, Rath $\mathrm{G}$, Morjani $\mathrm{H}$, et al: Interleukin-1betainduced apoptosis through adenylyl cyclase and ERK1/2 inhibition in primary cultured thyroid cells. Biochem Biophys Res Commun 339: 469-476, 2006.

23. Maio M, Coral S, Sigalotti L, et al: Analysis of cancer/testis antigens in sporadic medullary thyroid carcinoma: expression and humoral response to NY-ESO-1. J Clin Endocrinol Metab 88: 748-754, 2003.

24. Ruschenburg I, Kubitz A, Schlott T, et al: MAGE-1, GAGE-1/-2 gene expression in FNAB of classic variant of papillary thyroid carcinoma and papillary hyperplasia in nodular goiter. Int $\mathbf{J} \mathbf{M o l}$ Med 4: 445-448, 1999. 